\title{
A Case Series Assessing the Use of Xenon for Treatment of PTSD
}

\author{
V. Bogin', A. Dobrovosky²
}

${ }^{1}$ Nobilis Therapeutics, Inc., Portland, OR, ${ }^{2}$ Pirogov Russian National Research Medical University, Moscow, Russia

\section{ABSTRACT}

Current treatments of Post-traumatic Stress Disorder (PTSD) are limited by poor efficacy and adverse effects,. The established safety profile of subanesthetic concentrations of xenon gas, which is known to act as a glutamate subtype NMDA receptor antagonist, coupled with preclinical studies demonstrating its effects in an animal model, prompted us to evaluate its feasibility and efficacy in the treatment of patients with PTSD. A case series of ten patients with PTSD diagnosed with PCL-5 is presented here.

Several conclusions can be made: 1) xenon is a potentially effective modality in treatment of PTSD; 2) an anti-PTSD effect of xenon administration persists for at least two months after the completion of the active phase of treatment; 3) xenon inhalation is well tolerated. 4) xenon may be considered as an alternative to SSRIs in conjunction with cognitive-behavioral therapy as a safe modality in treatment of PTSD. These data support the need for randomized double-blind clinical trials to further study xenon-based interventions.

\section{Xenon Properties}

- Rare element (noble gas) present at $0.0000087 \%$ in air

- Used in humans for anesthesia, minimum alveolar concentration (MAC; 70\%) and as a contrast agent in diagnostic imaging $(28 \%)$

- Rapid on/off effects; has excellent safety/ side effect profiles and is well tolerated.

- Shown to be an antagonist of glutamate neurotransmission through blockade of NMDA receptors at the glycine co-agonist modulatory site (illustration, right).

- Shown to block reconsolidation of fear memory in an animal model of PTSD ${ }^{1}$

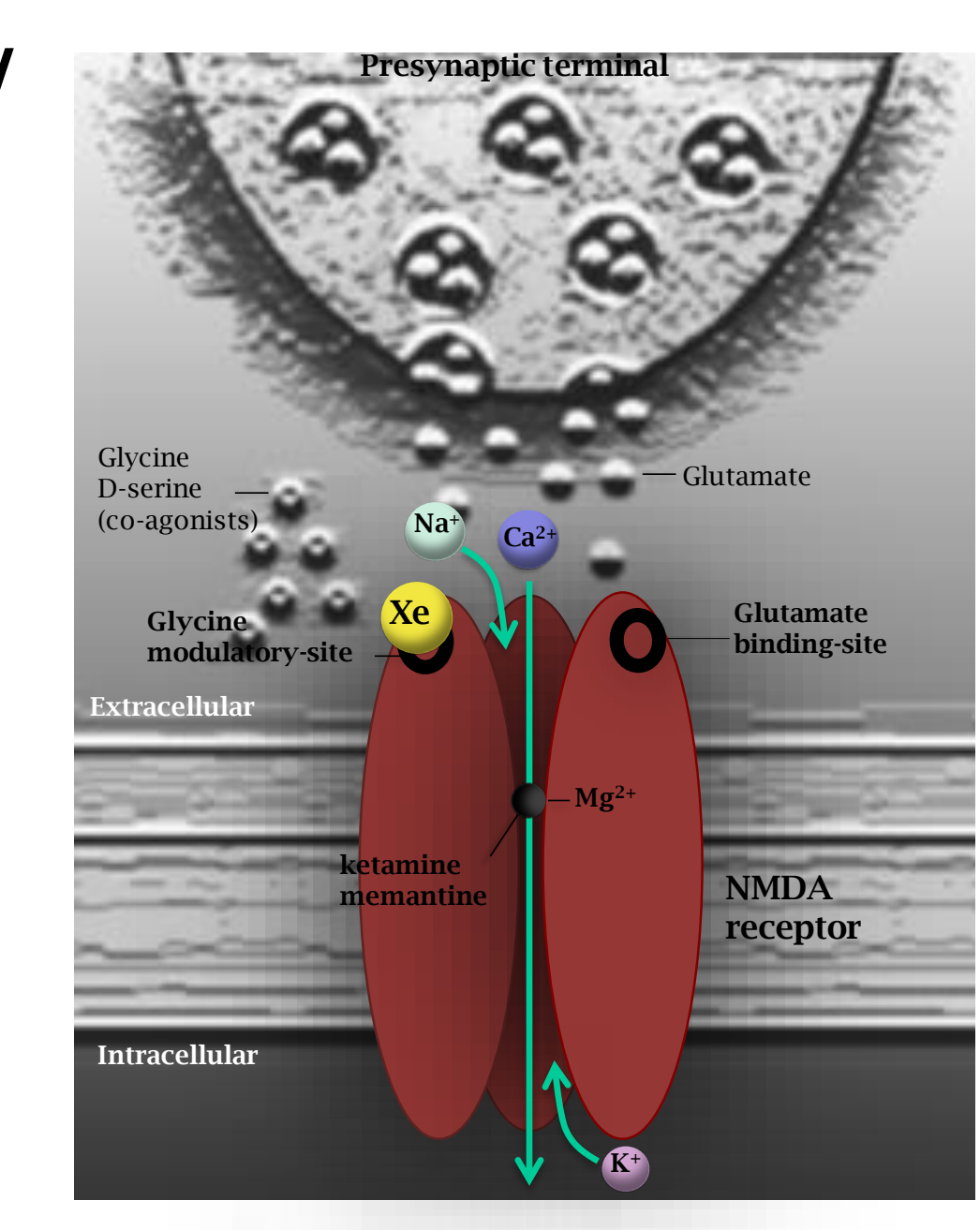

Ligand-gated ion channels are targets of xenon

\begin{tabular}{|c|c|c|c|c|}
\hline Receptor & Ligand & $\begin{array}{l}\text { Xenon } \\
\text { Action }\end{array}$ & $\begin{array}{l}\text { Effect on neuro } \\
\text { transmission }\end{array}$ & Reference \\
\hline NMDA & glutamate & $\begin{array}{c}\text { antagonist } \\
\text { (glycine site) }\end{array}$ & & 2 \\
\hline AMPA & glutamate & antagonist & & 3 \\
\hline $\begin{array}{c}\text { nACH }(\alpha 4 \beta 2>\alpha 4 \beta 4, \\
\alpha 7)\end{array}$ & acetylcholine & antagonist & & 4 \\
\hline $5-\mathrm{HT3}$ & serotonin & antagonist & & 5 \\
\hline GlyR1 & glycine & agonist & & 6 \\
\hline $\mathrm{GABA}_{A} \mathrm{R}$ & GABA & agonist & & 7 (yes); 8 (no) \\
\hline $\begin{array}{c}\text { TREK-1 channel } \\
\text { (two-pore domain } \mathrm{K}^{+} \text {channel) }\end{array}$ & $\begin{array}{l}\text { Temperature- osmo and } \\
\text { mechano-sensitive }\end{array}$ & activator & Hyperpolarize cell & 9 \\
\hline $\mathrm{K}_{\mathrm{ATP}}$ channel & ATP & channel opener & Hyperpolarize cell & 10 \\
\hline HCN2 channel & $\begin{array}{l}\text { Hyperpolarization } \\
\text { activated } \rightarrow \text { cAMP }\end{array}$ & inhibitor & Hyperpolarize cell & 11 \\
\hline
\end{tabular}

Other effects of xenon:

1) Increases brain derived neurotrophic factor (BDNF) $)^{12}$

2) Increases erythropoietin (EPO) levels ${ }^{13}$

3) Inhibits tissue plasminogen activator (tPA $)^{14}$

4) Alters pro- and anti-inflammatory factors ${ }^{15,16}$

5) Shown to have neuroprotective and anti-apoptotic effects ${ }^{17,18}$

\section{METHODS}

Ten patients ( 6 women and 4 men, mean age 39 years), with a diagnosis of "PTSD" (F43.10) according to ICD-10 were treated with xenon-containing gas mixture.

Administration of xenon was performed through inhalation of xenon-oxygen mixtures that were escalated from $15 \% / 85 \%$ to $30 \% / 70 \%$ with titration increments of $5 \%$ per session. Each patient in the study underwent between 6 and 7 treatments with xenon-oxygen mixture. The first three sessions were carried out daily and from session 4 onward - every other day. The selected dosing regime and the composition of the gas mixtures were based on the historical evidence of safety of subanesthetic use of xenon in imaging.

Table 2. Treatment Sessions and Corresponding Physiological Data

\begin{tabular}{|c|c|c|c|c|c|c|}
\hline & $\begin{array}{c}\text { Inhaled } \\
\text { Xenon/Oxygen } \\
(\%)\end{array}$ & $\begin{array}{l}\text { Amount of } \\
\text { Xenon used } \\
\text { (avg, L) }\end{array}$ & $\begin{array}{l}\text { Duration of } \\
\text { session } \\
\text { (avg, min) }\end{array}$ & $\begin{array}{c}\text { Vital signs } \\
\text { before script } \\
\text { (avg) }\end{array}$ & $\begin{array}{l}\text { Vital signs } \\
\text { after script } \\
\text { (avg) }\end{array}$ & $\begin{array}{c}\text { Vital signs } 20 \\
\text { min after } \\
\text { Xe/O2 (avg) }\end{array}$ \\
\hline Session 1 & $15 \% / 85 \%$ & 1.9 & 3 & $\begin{array}{l}\text { BP 126/78 HR } \\
74\end{array}$ & $\begin{array}{l}\text { BP 132/84 } \\
\text { HR } 99\end{array}$ & $\begin{array}{l}\text { BP 110/78 } \\
\text { HR } 66\end{array}$ \\
\hline Session 2 & $20 \% / 80 \%$ & 2.1 & 3.5 & $\begin{array}{l}\text { BP 128/76 HR } \\
\text { HR } 75\end{array}$ & $\begin{array}{l}\text { BP 131/80 } \\
\text { HR } 100\end{array}$ & $\begin{array}{l}\text { BP 111/74 } \\
\text { HR } 67\end{array}$ \\
\hline Session 3 & $25 \% / 75 \%$ & 2.3 & 3.5 & $\begin{array}{l}\text { BP 124/74 } \\
\text { HR } 72\end{array}$ & $\begin{array}{l}\text { BP } 128 / 78 \\
\text { HR } 98\end{array}$ & $\begin{array}{l}\text { BP 117/71 } \\
\text { HR } 61\end{array}$ \\
\hline Session 4 & $25 \% / 75 \%$ & 2.3 & 3.5 & $\begin{array}{l}\text { BP 126/74 HR } \\
\text { HR } 71\end{array}$ & $\begin{array}{l}\text { BP } 120 / 80 \\
\text { HR } 94\end{array}$ & $\begin{array}{l}\text { BP 112/76 } \\
\text { HR } 65\end{array}$ \\
\hline Session 5 & $25 \% / 75 \%$ & 2.5 & 3.8 & $\begin{array}{l}\text { BP 123/72 } \\
\text { HR } 74\end{array}$ & $\begin{array}{l}\text { BP 128/78 } \\
\text { HR } 95\end{array}$ & $\begin{array}{l}\text { BP 111/72 } \\
\text { HR } 63\end{array}$ \\
\hline Session 6 & $25 \% / 75 \%$ & 2.7 & 4 & $\begin{array}{l}\text { BP 128/76 } \\
\text { HR } 73\end{array}$ & $\begin{array}{l}\text { BP 134/78 } \\
\text { HR } 93\end{array}$ & $\begin{array}{l}\text { BP 107/72, } \\
\text { HR } 64\end{array}$ \\
\hline
\end{tabular}

Table 3: The results of averaged PCL-5 administered before the treatment (Visit 1), at the completion of the treatment (Visit 2) and during subsequent follow up (Visit 3)

\begin{tabular}{|c|c|c|c|}
\hline $\begin{array}{c}\text { Visit 1 } \\
\text { Day -14 to 1 }\end{array}$ & $\begin{array}{c}\text { Visit 2 } \\
\text { Day 21- 37 }\end{array}$ & $\begin{array}{c}\text { Visit 3 } \\
\text { Day 44- 62 }\end{array}$ & $\begin{array}{c}\text { Reduction Visit 1 to Visit 3 } \\
\text { (\%) }\end{array}$ \\
\hline 70 & 28 & 12 & 80.3 \\
\hline
\end{tabular}

CONCLUSIONS

These case series demonstrate remarkable improvement in 10 patients with PTSD symptoms. We believe that by combining xenon inhalation with trauma memory reactivation through a script-driven recounting of the event, memory reconsolidation was effectively inhibited. Given new evidence for the use of NMDA receptor antagonists as a promising treatment for PTSD, and emerging strategies that combine targeted medications with psychotherapy sessions, these case studies data firmly support further well-designed studies. 